\title{
The use of vagal manoeuvres in narrow complex tachyarrhythmias in primary care
}

Shane Murphy ( $\nabla$ murphy.shanedarren@gmail.com )

University of the Witwatersrand

Michele Torlutter

University of the Witwatersrand

\section{Method Article}

Keywords: family medicine, primary care, rural medicine, supraventricular tachycardia, vagal manoeuvre

Posted Date: December 22nd, 2021

DOI: https://doi.org/10.21203/rs.3.rs-1176226/v1

License: (9) This work is licensed under a Creative Commons Attribution 4.0 International License.

Read Full License

Version of Record: A version of this preprint was published at South African Family Practice on January 26th, 2022. See the published version at https://doi.org/10.4102/safp.v64i1.5413. 


\section{The use of vagal manoeuvres in narrow complex tachyarrhythmias in primary care}

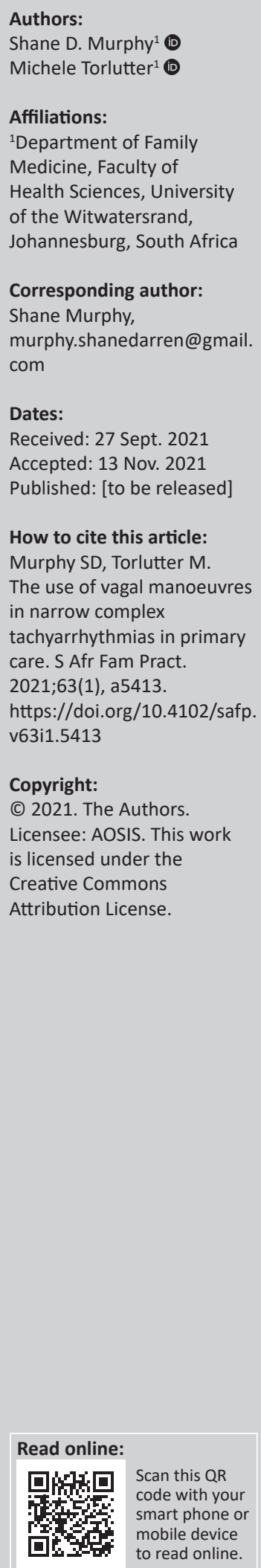

Supraventricular tachydysrhythmias (SVTs) are a common presenting complaint, with a national prevalence of $3 / 1000$ persons. Whilst most commonly stable, prolonged paroxysms can deteriorate into haemodynamically unstable subtypes or ventricular dysrhythmias. Early recognition with appropriate management is critical to reducing the morbidity associated with this condition. The American Heart Association holds that vagal manoeuvres are a first-line therapy in the management algorithm of stable SVTs. However, they state that no clear recommendations can be made around which manoeuvre to use, highlighting that future research should examine the efficacy and safety profiles of the various manoeuvres. In the South African primary care setting, clinicians must be at the forefront of pragmatic management strategies in the face of resource limitations, such as the unavailability of adenosine - a second-line therapy when vagal manoeuvres fail. In this article, we begin with a case study and review the literature around vagal manoeuvres.

Keywords: family medicine; primary care; rural medicine; supraventricular tachycardia; vagal manoeuvre.

\section{Case study Presentation}

A 35-year-old man presented to our centre during a weekend shift. He reports a 2-h history of experiencing palpitations and anxiety. The symptoms had begun at around 4 o'clock in the afternoon after a Sunday lunch with his family. He had drunk four units of alcohol over a 3-h period and smoked three cigarettes earlier that day.

\section{Past medical}

He reported two previous episodes at 29 and 33 years of age. During the previous episodes, inpatient workup had ruled out any metabolic and/or endocrinological abnormalities, as well as structural heart disease (by ECG and echocardiography). He is currently awaiting his cardiology outpatient appointment date. He has no chronic medical illnesses and had an appendectomy at 13 years of age. There was no family history of cardiac disease.

\section{Social}

He reports occasional alcohol use of around four units of alcohol per weekend, with no use during the week. He is not a regular smoker, smoking up to five cigarettes in a month. The patient did not use any illicit substances and drank three cups of coffee per day. He resides in a four-room house with his life partner of 6 years. Both are employed, have stable jobs, and he reports no psychosocial stressors.

\section{Examination}

The patient's observations were as follows:

- Blood pressure: 135/85

- Pulse: 215

- Saturation $96 \%$ (room air)

- Respiratory rate: 22

Examination reveals good peripheral perfusion with a regular tachycardia. Air entry was equal bilaterally, without adventitious sounds. There was no lower limb oedema or jugulovenous distension. The liver was not palpable. 


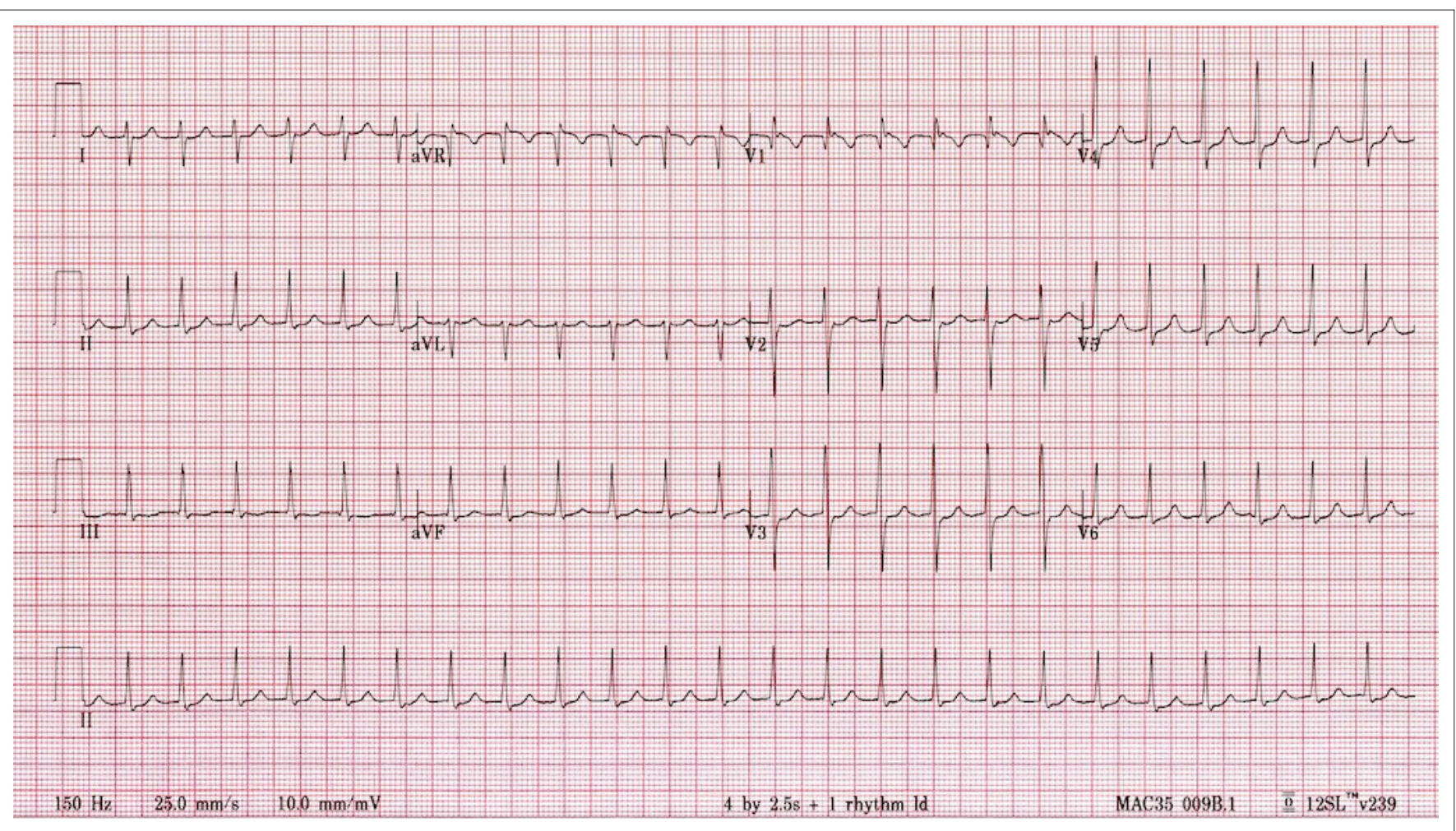

Source: Life in the Fast Lane; https://litfl.com/supraventricular-tachycardia-svt-ecg-library/

FIGURE 1: ECG example of narrow complex supraventricular tachydysrhythmias.
This patient was assessed as having a stable supraventricular tachycardia (SVT). He was moved to the high care area in the emergency centre, placed on monitors, baseline bloods were drawn (venous blood gas, FBC, UEC and CRP) and an ECG was done that showed a regular, narrow complex tachycardia.

A modified Valsalva manoeuvre was performed and normal sinus rhythm was restored. A post-reversion ECG was unremarkable.

\section{Background}

Tachydysrhythmias are defined as abnormal heart rhythms with a ventricular rate above 100 beats per minute. ${ }^{1}$ Supraventricular tachydysrhythmias are dysrhythmias that originate at, or above, the level of the His-Purkinje system ${ }^{2}$ from either atrial tissue or the atrioventricular (AV) junction:

- Atrial tissue

- Sinus tachycardia

- Inappropriate sinus tachycardia

- Sinoatrial nodal re-entrant tachycardia (SNRT)

- Atrial fibrillation

- Atrial flutter

- Intra-atrial re-entrant tachycardia

- Atrioventricular junction

- Atrioventricular nodal re-entrant tachycardia (AVNRT)

- Atrioventricular re-entrant tachycardia (AVRT)

- Junctional tachycardia

Supraventricular tachydysrhythmias are most commonly narrow complex tachydysrhythmias but can have broad complexes when there is pre-existing aberrant conduction (e.g. bundle branch blocks), tachycardia-induced aberrancy (physiological), or, in the case of an accessory pathway, with antidromic conduction.

Whilst local literature on the incidence of SVT is sparse, evidence shows that the national prevalence approximates international figures at around 3/1000 persons. ${ }^{3}$ The majority of SVTs are because of re-entry pathways, without the presence of underlying cardiac disease. ${ }^{2}$ Common symptoms include syncope/presyncope, palpitations, shortness of breath, diaphoresis, chest pain and lightheadedness/dizziness.

\section{An approach}

Most cases of SVT are stable unless there is an underlying cardiac failure, ischaemia or other co-morbidities (such as sepsis or toxin ingestion).

Determining the haemodynamic stability of the patient is the first step in evaluation. A useful mnemonic for this is HASIA:

- Hypotension

- Altered mentation

- Shock

- Ischaemic chest pain (poor coronary perfusion pressure)

- Acute pump failure (pulmonary oedema)

If any of these cues are present, attempt to rapidly rule out sinus tachycardia ${ }^{2}$ (and treat the underlying cause). If this is ruled out, immediate synchronised cardioversion according to Advanced Cardiac Life Support principles is warranted. 
Provided there are no emergent signs or symptoms that warrant synchronised cardioversion, we regard the SVT as stable and proceed with the evaluation of the SVT2:

- Is it regular or irregular?

- Are P waves discernible? If so, what is their morphology?

\section{Perform the Valsalva maneuver!!:}

The American Heart Association describes vagal manoeuvres as a class one recommendation (level of evidence B-R) for first-line therapy of stable SVTs, 2 although they note that vagal manoeuvres require further refinement to establish their effectiveness and safety. 2

\section{Which vagal manoeuvre is best?}

Studies have recurrently shown the Valsalva manoeuvre $(\mathrm{VM})$ as superior to other options in adults:

- Valsalva success rate: $20 \%-55 \% 0^{4,5}$

- Carotid sinus massage success rate: $5 \%-33 \%{ }^{5,6}$

- Diving reflex (cold ice immersion) success rate: $17 \%{ }^{7}$

The diving reflex can be technically challenging and is not frequently employed in adults. In children, eliciting the diving reflex by use of a cold water bucket is supported as a first choice intervention by high quality evidence. ${ }^{2}$ Whilst the carotid sinus massage is the simplest to perform, studies have reported iatrogenic peril (thromboembolic stroke from dislodged carotid atheroma) in around 1\% of cases. ${ }^{6}$ The greater effectiveness and improved risk profile of the VM have seen it become a first choice vagal manoeuvre for the treatment of stable SVT in adult patients.

BOX1: What about adenosine?

\section{The pharnacolnetics of adesosine}

The pharmacokinetics of adenosine make it a highly favourable agent $\mathrm{t}^{4}$ :

- Rapid onset

- Short half-life (<10 seconds)

- Highly selective for adenosine-1 receptor:

A1 receptor - induces asystole by inhibiting adenylyl cyclase and reducing

intracellular CAMP, which hyperpolarizes cardiac cells by increasing K+ efflux intracellular CAMP, which hyperpolarizes cardiac
(in turn inhibiting Ca2+ and $B$-agonist receptors)

- Rapid intracellular metabolism

However, the adverse effects of adenosine include a high incidence of new and transient cardiac arrhythmia (up to $55 \%)$, dyspnoea $(12 \%-28 \%)$, chest tightness $(40 \%)$, dizziness $(<12 \%)$, headache, facial flushing $(44 \%)$, nausea and an 'electric shock' sensation.

Contraindications include hypersensitivity, high-grade heart block, sick sinus rhythm, bronchoconstrictive or bronchospastic disease, including asthma. ${ }^{5}$

The success of adenosine is dependent on several technical factors such as the proximity of venous access to heart, elevation of the limb and the rapid use of saline flush with a three-way stop cock.

\section{Source:}

Note: In South Africa, adenosine is not available at community health centres or clinics. It is not found in the essential drug list and is not mentioned as a step in the management of tachydysrhythmias in the standard treatment guidelines. ${ }^{6}$
A recent modification to the $\mathrm{VM}$ was employed in the landmark REVERT trial, ${ }^{8}$ which showed increased sinus reversion rates (compared to the conventional VM) and no increase in harm.

Phase I:

- Increased intrathoracic pressure - SBP rises $(>15 \mathrm{mmHg})$ following initiation of Valsalva (10 s)

- Triggers baroreceptor reflex (either at a carotid bifurcation or within aortic arch)

- Afferent impulses via glossopharyngeal (carotid) or vagus (aortic) nerve to vagal nuclei within nucleus tractus solitarius within the medulla

Phase II:

- Decreased venous return; increased systemic arterial pressure; blood pressure return to (just below) normal whilst a strain is maintained

- Efferent impulses from vagal nuclei descend both right and left vagal nerves to heart, lungs and gastrointestinal tract.

- Right vagus nerves stimulate sinoatrial (SA) node decreased firing

TABLE 2: The Revert trial-Frequency of adverse events.

\begin{tabular}{lcc}
\hline $\mathbf{x x x}$ & Standard VM $(\boldsymbol{n}=\mathbf{2 1 4})$ & Modified VM $(\boldsymbol{n}=\mathbf{2 1 4})$ \\
\hline Increased heart rate & 4 & 3 \\
Hypotension or light-headedness & 3 & 3 \\
Electrocardiograph captured events & 2 & 3 \\
Other $\dagger$ & 0 & 5 \\
Musculoskeletal pain $\dagger$ & 0 & 4 \\
\hline
\end{tabular}

Source: Adapted from Appelboam et al. ${ }^{8}$

VM, Valsalva manoeuvre.

$\dagger$, Transient headache $(n=2)$; shortness of breath $(n=1)$; cyanosis $(n=1)$ in different patients; $\$$, Transient chest wall pain on straining.

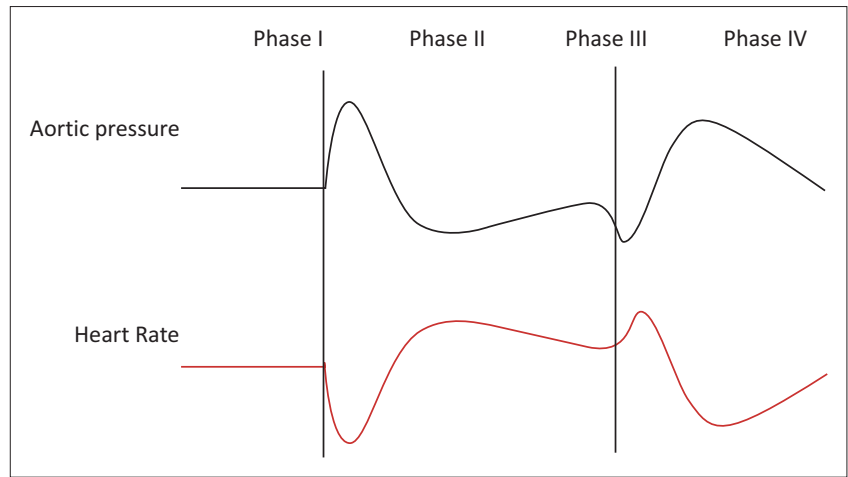

Source: Adapted: Wen et al.'?

FIGURE 2: The physiology of the Valsalva manoeuvre.

TABLE 1: The REVERT trial - Primary and secondary outcomes.

\begin{tabular}{|c|c|c|c|c|c|c|c|}
\hline \multirow[t]{2}{*}{$\mathbf{x x x}$} & \multicolumn{2}{|c|}{ Standard VM } & \multicolumn{2}{|c|}{ Modified VM } & \multicolumn{2}{|c|}{ Effect size } & \multirow[t]{2}{*}{$p$} \\
\hline & $n=214$ & $\%$ & $n=214$ & $\%$ & $\mathbf{x x}$ & $95 \% \mathrm{Cl}$ & \\
\hline Presence of sinus rhythm at $1 \mathrm{~min}$ & 37 & 17 & 93 & 43 & 3.7 & $2.3-5.8$ & $<0.0001$ \\
\hline Adenosine needed & 148 & 69 & 108 & 50 & 0.45 & $0.30-0.68$ & 0.0002 \\
\hline Any other emergency anti-arrhythmic needed & 171 & 80 & 121 & 57 & 0.33 & $0.21-0.51$ & $<0.0001$ \\
\hline Discharged home from emergency room & 146 & 68 & 134 & 63 & 0.79 & $0.51-1.21$ & 0.28 \\
\hline Any adverse events & 8 & 4 & 13 & 6 & 1.61 & $0.63-4.08$ & 0.32 \\
\hline
\end{tabular}


- Left vagus innervates av node - decreased conduction Phase III:

- Decreased intrathoracic pressure. Blood pressure drops when strain is released.

Phase IV:

[AQ14] - Rapid increase in BP as sympathetic surge response to decreased systolic pressure.

- The response to the hypotension induced by the VM is the final means by which we induce a strong vagal tone to terminate the tachydysrhythmia.

The purpose of a vagal manoeuvre is to impact cardiac physiology. The desired effects are mostly achieved in phases I and IV $^{9}$ :

- SA node - slowed impulse generation in SA node.

- Atria - normal conduction velocity; prolonged refractory period.

- AV node - decreased conduction in AV node with prolonging of the refractory period.

- Ventricles - decreased inotropy; normal conduction in His-Purkinje system.

When should we use the VM?

- Therapeutic - Haemodynamically stable patients: firstline therapy of SVT.

- Diagnostic - May help distinguish SVT from VT by slowing conduction.

As mentioned, the most profound vagotonic effects are induced at the SA and AV nodes, and hence these rhythms undergo the highest reversion success rates:

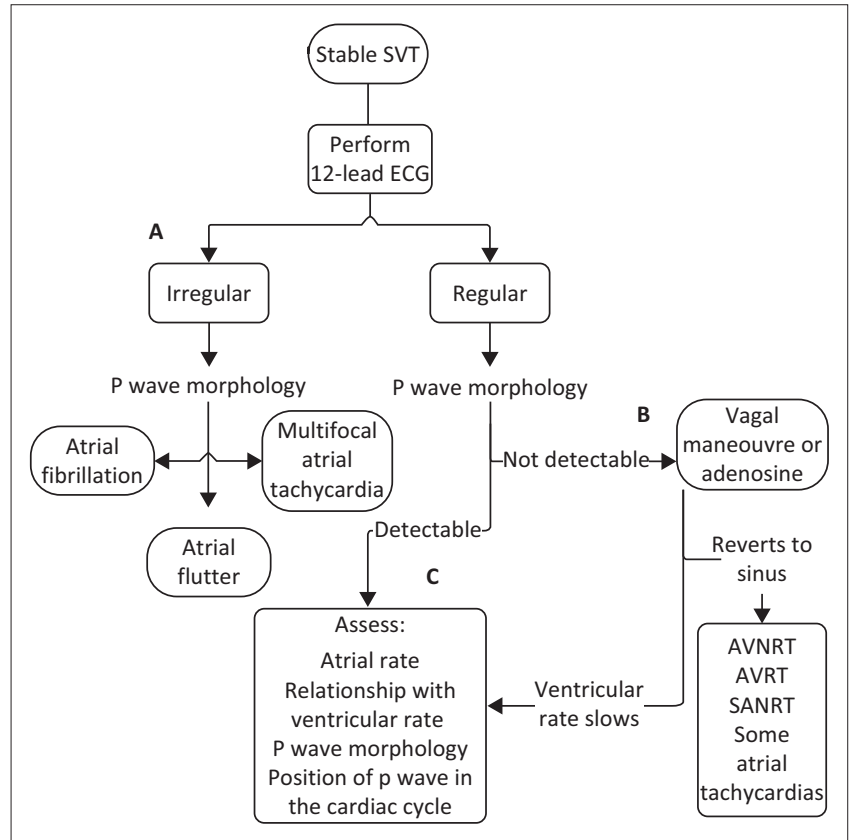

Source: Adapted from Richard et al. ${ }^{2}$

ECG, xxx; AVNRT, atrioventricular nodal re-entrant tachycardia; AVRT, atrioventricular [AQ15] re-entrant tachycardia; SANRT, xxx; SVT, supraventricular tachydysrhythmias.

FIGURE 3: Evaluation of supraventricular tachydysrhythmias.
- A - Vagal manoeuvres can terminate tachycardias of atrial origin, but they have a lower success rate and care must be taken to not perform AV node blockade in wide-complex tachycardias or if the patient has a known accessory pathway (risk of inducing ventricular fibrillation).

- B - Vagal manoeuvres are most successful in AV nodaldependent SVTs (AVRT and AVNRT). At this stage, the clear dichotomy between therapeutic effect (reversion to sinus rhythm) and diagnostic application (slowing the rate to discern the dysrhythmia present and determine the best management approach) can be noted.

- C - If $\mathrm{p}$ waves are present and can be characterised, either can vagal manoeuvres be tried or an effective therapeutic agent selected based on the type of rhythm.

\section{How to perform the modified Valsalva ${ }^{8}$}

$[\mathrm{AQ16}]$

Equipment:

- 12 lead EKG placed on patient

- Cardiac monitoring - continuous

- Pulse oximetry

- Non-invasive BP monitoring - cycling frequently

- Intravenous access with fluids either running or immediately available

- Resuscitation trolley available

- Airway

- Oxygen

- Crash cart with appropriate antiarrhythmic available +- procedural sedation for cardioversion

Staff:

- Lead clinician

- Nursing assistant for monitoring

- + Additional assistant to elevate legs

[AQ18]

An informed, cooperative patient is paramount. The American Heart Association advocates teaching a patient to cough on command (vagotony from coughing terminates a bradycardia consequent to the Valsalva). ${ }^{2}$

Technique:

- Attach all monitors, have support staff.

- Patient is seated in an upright seated position.

- Patient blows against a $10 \mathrm{~mL}$ syringe to move plunger (approximately 30-40 mmHg) for 10-20 s

- Immediately drop patient to supine position

- Simultaneously elevate patient's legs to $45^{\circ}-90^{\circ}$

- Hold for 45-60 s

- Repeat if necessary

The 'modification' of the Valsalva by dropping the head of the patient, elevating the legs, and finally lifting the head of the patient accentuates the physiological effects of the Valsalva manoeuvre - increasing the likelihood of success.

\section{Clinical bottom line and applicability to the patient}

Supraventricular tachydysrhythmias are the most common tachyarrhythmias with an incidence of 3/1000 persons. Most 
SVTs are benign conditions without underlying structural heart pathology. However, their propensity to deteriorate into unstable SVTs or ventricular fibrillation mandates early and effective treatment. The modified VM is the most effective vagal manoeuvre with minimal documented adverse effects and should be used as the first-line therapy in the management of SVT.

The patient in our case study was a 35-year-old man who presented with a recurrent, stable AVNRT in the absence of any underlying structural heart disease. The effective management of his AVNRT avoided unnecessary admission to a higher level of care whilst awaiting his outpatient cardiology appointment. This case highlights the value of evidence-based practice in the primary care setting to provide appropriate care whilst avoiding unnecessary medical intervention with its associated costs and potential harm.

\section{Acknowledgements Competing interests}

The authors declare that they have no financial or personal relationships that may have inappropriately influenced them in writing this article.

\section{Authors' contributions}

S.D.M. was responsible for conceptualisation and the original draft. M.T. was responsible for review and editing.

\section{Ethical considerations}

This article followed all ethical standards for research without direct contact with human or animal subjects.

\section{Funding information}

This research received no specific grant from any funding agency in the public, commercial or not-for-profit sectors.

\section{Data availability}

Data sharing is not applicable to this article as no new data were created or analysed in this study.

\section{Disclaimer}

The views and opinions expressed in this article are those of the authors and do not necessarily reflect the official policy or position of any affiliated agency of the authors.

\section{References}

1. Lindberg DM. Modified Valsalva maneuver for Supraventricular Tachycardia. NEJM J Watch Emerg Med Walth [serial online]. 2015 [cited 2019 Apr 3]. Available from: https://search.proquest.com/docview/1707983466/abstract/941C07D98 $\mathrm{BA} 84 \mathrm{E} 65 \mathrm{PQ} / 21$

2. Page RL, Joglar JA, Caldwell MA, et al. ACC/AHA/HRS guideline for the management of adult patients with supraventricular tachycardia. Circulation. 2016;133(14): e506-e574. https://doi.org/10.1161/CIR.0000000000000311

3. Bonny A, Ngantcha $M$, Scholtz $W$, et al. Cardiac arrhythmias in Africa. J Am Coll Cardiol. 2019;73(1):100-109. https://doi.org/10.1016/j. jacc.2018.09.084

4. Smith G. Management of supraventricular tachycardia using the Valsalva manoeuvre: A historical review and summary of published evidence. Eur J Emerg Med. 2012;19(6):346-352. https://doi.org/10.1097/MEJ.0b013e3 2834ec7ad

5. Lim SHC, Lai SM, Wong KCK. Head down deep breathing for cardioversion of paroxysmal supraventricular tachycardia. Case Rep Emerg Med. 2018;2018:1-3. https://doi.org/10.1155/2018/1387207

6. Collins NA, Higgins III GL. Reconsidering the effectiveness and safety of carotid sinus massage as a therapeutic intervention in patients with supraventricular tachycardia. Am J Emerg Med. 2015;33(6):807-809.

7. Wen ZC, Chen SA, Tai CT, Chiang CE, Chiou CW, Chang MS. Electrophysiological mechanisms and determinants of vagal maneuvers for termination of paroxysmal supraventricular tachycardia. Circulation. 1998;98(24):2716-2723. https://doi. org/10.1161/01.CIR.98.24.2716

8. Appelboam A, Reuben A, Mann C, et al. Postural modification to the standard Valsalva manoeuvre for emergency treatment of supraventricular tachycardias (REVERT): A randomised controlled trial. Lancet. 2015;386(10005):1747-1753. https://doi.org/10.1161/01.CIR.98.24.2716

9. Pstras L, Thomaseth K, Waniewski J, Balzani I, Bellavere F. The Valsalva manoeuvre: Physiology and clinical examples. Acta Physiol Oxf Engl. 2016;217(2):103-119. https://doi.org/10.1111/apha.12639

10. Pallin DJ. Modified Valsalva maneuver increases conversion of paroxysma supraventricular tachycardia. NEJM J Watch Emerg Med Walth [serial online] 2017 [cited 2019 Apr 3]; Available from: https://search.proquest.com/docview/ 1907733598/abstract/941C07D98BA84E65PQ/25 


\section{Figures}

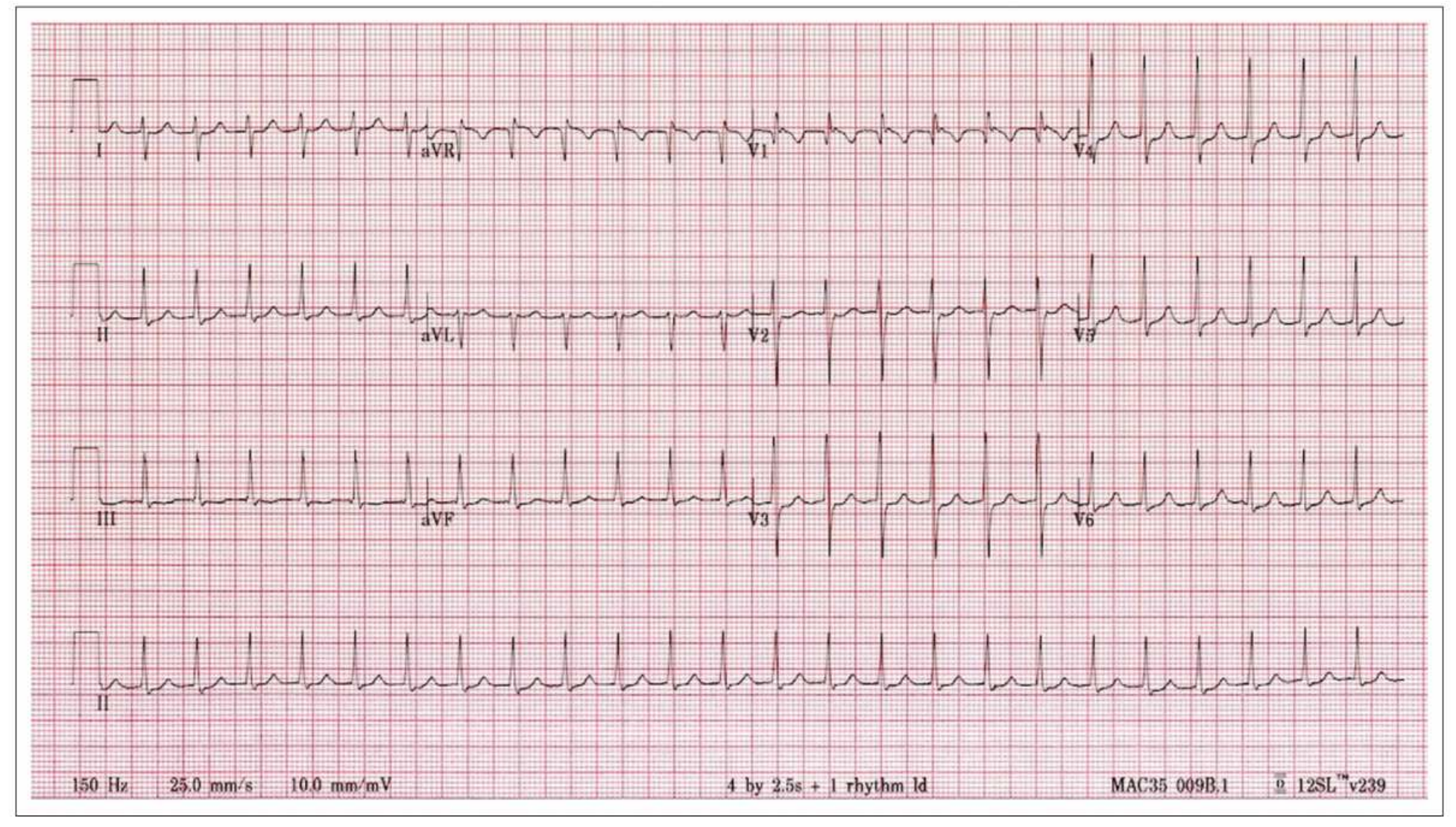

Figure 1

ECG example of narrow complex supraventricular tachydysrhythmias. 
Figure: The physiology of the valsalva, manoeuvre (adapted: Wen et al., 1998) ${ }^{10}$

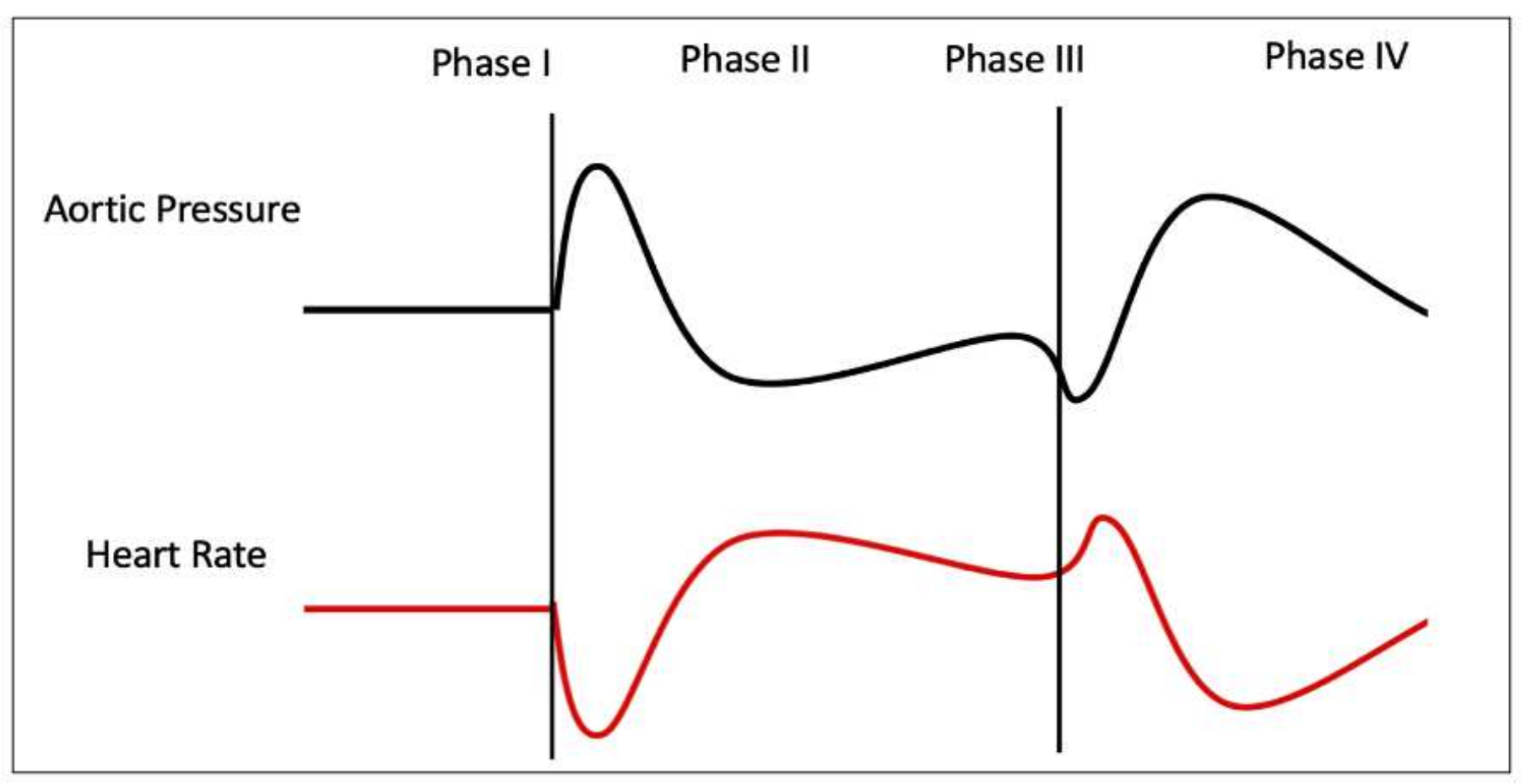

Figure 2

The physiology of the Valsalva manoeuvre. 


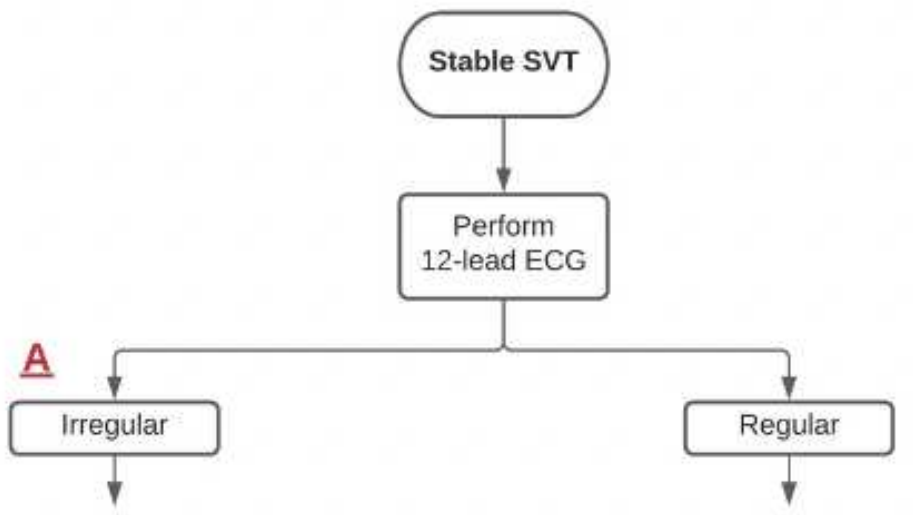

P Wave Morphology

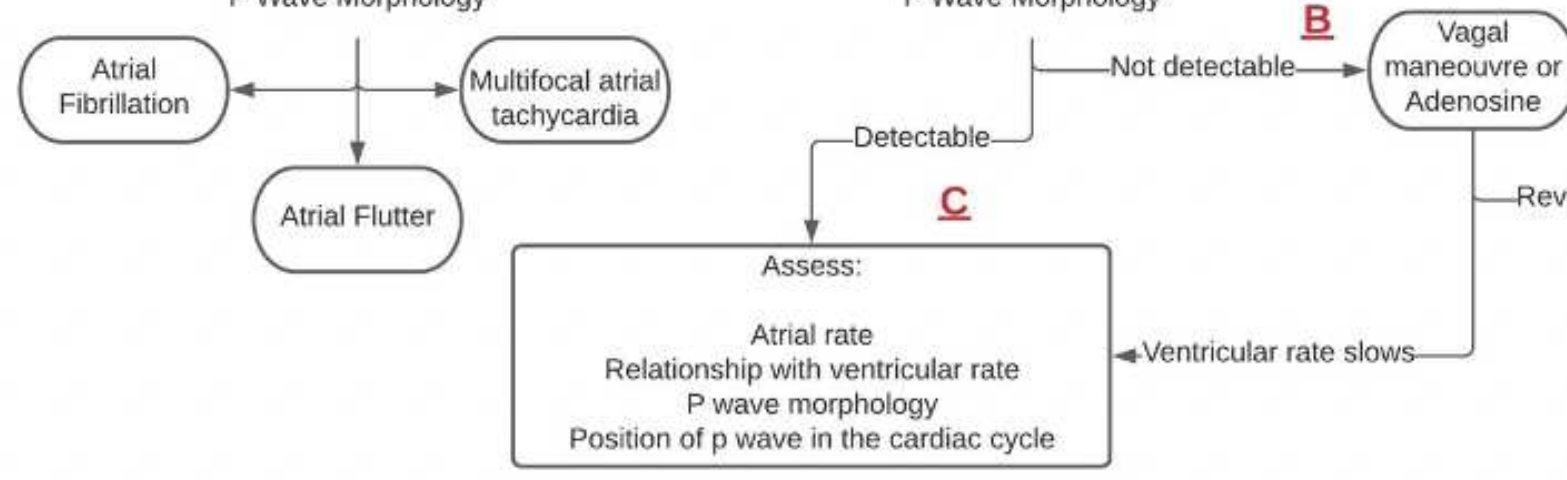

to sinus

AVNRT
AVRT
SANRT
Some atrial
tachycardias

\section{Figure 3}

Evaluation of supraventricular tachydysrhythmias. 


\section{Box: What about Adenosine?}

The pharmacokinetics of adenosine make it a highly favourable agent: ${ }^{4}$

- Rapid onset

- Short half-life (<10 seconds)

- Highly selective for Adenosine-1 receptor

A1 receptor-induces asystole by inhibiting adenylyl cyclase and reducing intracellular CAMP which hyperpolarizes cardiac cells by increasing $\mathrm{K}+$ efflux (in turn inhibiting ca2+ and B-agonist receptors) ${ }^{4}$

- Rapid intracellular metabolism

However, the adverse experiences of adenosine Include a high incidence of new and transient cardiac arrhythmia (up to 55\%), dyspnoea (12-28\%), chest tightness (40\%), dizziness $(<12 \%)$, headache, facial flushing $(44 \%)$, nausea and an "electric shock" sensation. ${ }^{5}$

Contraindications include hypersensitivity, high grade heart block, sick sinus rhythm, broncho-constrictive or bronchospastic disease, including asthma. ${ }^{5}$

The success of adenosine is dependent on several technical factors such as proximity of venous access to heart, elevation of the limb, use of saline flush with three way stop cock.

* In South Africa, adenosine is not available at community health centres or clinics, it is not found in the essential drug list and not mentioned as a step in the management of tachydysrhythmias in the standard treatment guidelines. ${ }^{6}$

\section{Figure 4}

\section{Box 1: What about adenosine?}

